\title{
$H$-Decompositions of $r$-graphs when $H$ is an $r$-graph with exactly 2 edges
}

\author{
Teresa Sousa * \\ Departamento de Matemática, FCT-UNL and CMA-UNL \\ Quinta da Torre, 2829-516 Caparica, Portugal \\ tmjs@fct.unl.pt \\ Submitted: Dec 15, 2008; Accepted: Mar 1, 2010; Published: Mar 8, 2010 \\ Mathematics Subject Classification: 05C88
}

\begin{abstract}
Given two $r$-graphs $G$ and $H$, an $H$-decomposition of $G$ is a partition of the edge set of $G$ such that each part is either a single edge or forms a graph isomorphic to $H$. The minimum number of parts in an $H$-decomposition of $G$ is denoted by $\phi_{H}^{r}(G)$. By a 2-edge-decomposition of an $r$-graph we mean an $H$-decomposition for any fixed $r$-graph $H$ with exactly 2 edges. In the special case where the two edges of $H$ intersect in exactly 1,2 or $r-1$ vertices these 2-edge-decompositions will be called bowtie, domino and kite respectively. The value of the function $\phi_{H}^{r}(n)$ will be obtained for bowtie, domino and kite decompositons of $r$-graphs.
\end{abstract}

\section{Introduction}

An hypergraph is a (finite) set $V=V(G)$, called the vertices of $G$ together with a set $E=E(G)$ of non-empty subsets (of any cardinality) of $V$, called the hyperedges or edges. When all the edges of an hypergraph are distinct we say that the hypergraph is simple. If in addition all the edges have the same cardinality $r \geqslant 2$ then $G$ is said to be a uniform hypergraph or an $r$-graph. Thus, graphs are special hypergraphs. The number of vertices of an hypergraph is its order and is denoted by $v(G)$. The number of edges in an hypergraph is its size and is denoted by $e(G)$. The complete $r$-graph on $n$ vertices is the hypergraph that consists of all $r$-subsets of $V$ and it will be denoted by $K_{n}^{r}$. We denote by $[n]$ the set of the first $n$ integers.

Given two $r$-graphs $G$ and $H$, with $r \geqslant 2$, an $H$-decomposition of $G$ is a partition of the edge set of $G$ such that each part is either a single edge or forms a graph isomorphic to $H$. The minimum number of parts in an $H$-decomposition of $G$ is denoted by $\phi_{H}^{r}(G)$.

${ }^{*}$ This work was partially supported by Financiamento Base 2009 ISFL-1-297 from FCT/MCTES/PT 
For $r \geqslant 2$, we are interested in the value of the function

$$
\phi_{H}^{r}(n)=\max \left\{\phi_{H}^{r}(G) \mid v(G)=n\right\},
$$

which is the smallest number such that any $r$-graph $G$ of order $n$ admits an $H$-decomposition with at most $\phi_{H}^{r}(n)$ parts.

In the special case of $r=2$, i.e., when we have graphs, the asymptotic value of the function $\phi_{H}^{2}(n)$, simply denoted by $\phi_{H}(n)$, was obtained by Pikhurko and Sousa [3] for all graphs $H$. However, the value of the function $\phi_{H}^{r}(n)$ is still open for $r \geqslant 3$. We will present some results about the value of the function $\phi_{H}^{r}(n)$ for some special cases of $H$.

By a 2-edge-decomposition of an $r$-graph we mean an $H$-decomposition for any fixed $r$-graph $H$ with exactly 2 edges. In the special case where the two edges of $H$ intersect in exactly 1,2 or $r-1$ vertices these 2-edge-decompositions will be called bowtie, domino and kite respectively. In this paper the 2-edge-decomposition problem is solved asymptotically and we will also obtain the exact value $\phi_{H}^{r}(n)$ for some special cases of 2-edge-decompositions.

\section{2-Edge-Decompositions of $r$-Graphs}

We start this section with the following simple result.

Lemma 2.1. For any non-empty $r$-graph $H$ with $m$ edges and any integer $n$, we have

$$
\phi_{H}^{r}(n) \leqslant \frac{1}{m}\left(\begin{array}{l}
n \\
r
\end{array}\right)+\frac{m-1}{m} \operatorname{ex}(n, r, H),
$$

where $\operatorname{ex}(n, r, H)$ denotes the maximum number of edges that an $r$-graph with $n$ vertices can have without containing a copy of $H$.

Proof. To prove (2.1) remove greedily one by one the edge-sets of $H$-subgraphs of a given $r$-graph $G$ and then remove the remaining edges. The bound (2.1) follows as at most ex $(n, r, H)$ parts are single edges.

Let $G$ be an $r$-graph with vertex set $V(G)$ and edge set $E(G)$. We say that $\mathrm{G}$ is $t$-colorable if there is a map

$$
c: V(G) \rightarrow\{1, \ldots, t\}
$$

such that no edge $X \in E(G)$ in monochromatic.

Let $H$ be a fixed $r$-colorable $r$-graph with $\mathrm{m}$ edges. Erdős [2] proved that

$$
\operatorname{ex}(n, r, H)=o\left(n^{r}\right) \text {. }
$$

We have,

$$
\phi_{H}^{r}(n)=\left(\frac{1}{m}+o(1)\right)\left(\begin{array}{l}
n \\
r
\end{array}\right) .
$$


The upper bound in (2.2) follows directly from (2.1) and the result of Erdös [2] and the lower bound follows from $\phi_{H}^{r}(n) \geqslant \phi_{H}^{r}\left(K_{n}^{r}\right) \geqslant \frac{1}{m}\left(\begin{array}{l}n \\ r\end{array}\right)$.

Observe that the 2-edge-decomposition problem is asymptotically solved in (2.2) for $r$-colorable $r$-graphs.

In this section we will find the exact value $\phi_{H}^{r}(n)$ for some special cases of 2-edgedecompositions. Let $H$ be a fixed $r$-graph with exactly 2 edges. We will start by proving that $\phi_{H}^{r}(n) \leqslant \phi_{H}^{r}\left(K_{n}^{r}\right)$. Thus, it suffices to study 2-edge-decompositions of complete $r$ graphs. Then, we will also prove that the trivial lower bound

$$
\phi_{H}^{r}\left(K_{n}^{r}\right) \geqslant\left\lceil\frac{1}{2}\left(\begin{array}{l}
n \\
r
\end{array}\right)\right\rceil
$$

is in fact optimal for bowtie, domino and kite decompositions of $r$-graphs, for some values of $r$.

Definition 2.2. Let $H$ be a fixed r-graph with 2 edges and $\mathcal{F}$ an $H$-decomposition of an $r$-graph $G$. We say that $\mathcal{F}$ is optimal if it contains exactly $\phi_{H}^{r}(G)$ elements.

Theorem 2.3. Let $H$ be a fixed $r$-graph with 2 edges and $G$ an $r$-graph with $n$ vertices. Then,

$$
\phi_{H}^{r}(G) \leqslant \phi_{H}^{r}\left(K_{n}^{r}\right) .
$$

Proof. Let $\mathcal{F}$ be an optimal $H$-decomposition of $K_{n}^{r}$. We will extract from $\mathcal{F}$ an $H$ decomposition of $G, \mathcal{C}$, having at most $|\mathcal{F}|$ elements.

Let $F$ be an element of $\mathcal{F}$. If $F$ has two edges and both are edges of $G$ then we add $F$ to $\mathcal{C}$. If exactly on edge of $F$ is an edge of $G$ then add that single edge to $\mathcal{C}$. If the edges of $F$ are not edges of $G$ we discard $F$. After every element of $\mathcal{F}$ has been considered, the set $\mathcal{C}$ is clearly an $H$-decomposition of $G$ and we have

$$
\phi_{H}^{r}(G) \leqslant|\mathcal{C}| \leqslant|\mathcal{F}|=\phi_{H}^{r}\left(K_{n}^{r}\right),
$$

as required.

Theorem 2.3 implies that, when $H$ has exactly 2 edges, it suffices to study $H$-decompositions of complete $r$-graphs. In the sequel, and for the sake of simplicity, the edges of an $r$-graph will be written as $x_{1} \cdots x_{r}$ instead of $\left\{x_{1}, \ldots, x_{r}\right\}$.

\subsection{Bowtie Decomposition of the Complete $r$-Graph}

Theorem 2.4. Let $r=3$ and $n \geqslant 5$ or $4 \leqslant r \leqslant \frac{1}{6} \sqrt{n}$. Then any optimal bowtie decomposition of the complete r-graph of order $n$ has $\left\lceil\frac{1}{2}\left(\begin{array}{l}n \\ r\end{array}\right)\right\rceil$ elements.

Before proving the theorem we will need to introduce the tools. 
Theorem 2.5 (Dirac 1952, [1]). Every graph with $n \geqslant 3$ vertices and minimum degree at least $n / 2$ has a hamiltonian cycle.

Theorem 2.6 (Tutte 1947, [1]). A graph $G$ has a 1-factor (perfect matching) if and only if odd $(G-S) \leqslant|S|$ for all $S \subseteq V(G)$, where odd $(G-S)$ denotes the number of odd components of the graph $G-S$.

Theorem 2.7 (Ray-Chaudhuri,Wilson 1975, [4]). Let L be a set consisting of $s$ nonnegative integers and let $G$ be an $r$-graph with $n$ vertices. If $|A \cap B| \in L$ for any distinct edges $A, B \in E(G)$, then $e(G) \leqslant\left(\begin{array}{l}n \\ s\end{array}\right)$.

Proof of Theorem 2.4. The lower bound follows immediately, thus it suffices to find a bowtie decomposition of $K_{n}^{r}$ with $\left\lceil\frac{1}{2}\left(\begin{array}{l}n \\ r\end{array}\right)\right\rceil$ elements. Let $[n]$ be the vertex set of $K_{n}^{r}$. Let $G_{r}$ be the graph with $V\left(G_{r}\right)=E\left(K_{n}^{r}\right)$ and $E\left(G_{r}\right)=\left\{u v \mid u, v \in E\left(K_{n}^{r}\right)\right.$ and $\left.|u \cap v|=1\right\}$. Then, the theorem follows if we prove that $G_{r}$ has a Hamiltonian cycle or a matching that saturates at least $v\left(G_{r}\right)-1$ vertices.

Note that $G_{r}$ is a connected $r\left(\begin{array}{c}n-r \\ r-1\end{array}\right)$-regular graph. For $r=3$ and $n=5,6$ we can see by inspection that $G_{3}$ contains a perfect matching. If $r=3$ and $n \in\{7, \ldots, 11\}$ then Theorem 2.5 holds so $G_{3}$ has a Hamiltonian cycle. Suppose that $r=3$ and $n \geqslant 12$ or $4 \leqslant r \leqslant \frac{1}{6} \sqrt{n}$. In this case we will see that Tutte's Theorem holds. If necessary remove one vertex from $V\left(G_{r}\right)$ so that we have $v\left(G_{r}\right)$ even.

Definition 2.8. Let $G$ be an $r$-graph. The independence number of $G$, denoted by $\alpha(G)$, is the maximum number of pairwise non adjacent vertices of $G$.

Claim 1. $\alpha\left(G_{r}\right) \leqslant\left(\begin{array}{c}n \\ r-1\end{array}\right)$.

Proof. Let $\mathcal{A} \subseteq V\left(G_{r}\right)$ be an independent set. Then, $|A \cap B| \in\{0,2, \ldots, r-1\}$ for all distinct $A, B \in \mathcal{A}$. Considering the $r$-graph $([n], \mathcal{A})$, we have $|\mathcal{A}| \leqslant\left(\begin{array}{c}n \\ r-1\end{array}\right)$ by Theorem 2.7.

Claim 1 implies that for every $S \subseteq V\left(G_{r}\right)$ we have odd $\left(G_{r}-S\right) \leqslant\left(\begin{array}{c}n \\ r-1\end{array}\right)$. The following claim completes the proof of the theorem.

Claim 2. $|S| \geqslant \operatorname{odd}\left(G_{r}-S\right)$, for all $S \subseteq V\left(G_{r}\right)$.

Proof. Let $S \subseteq V\left(G_{r}\right)$. If $S=\emptyset$ then there is nothing to prove since $G_{r}$ is connected. Assume that $S \neq \emptyset$. Let $F_{1}, \ldots, F_{t}$ be the components of $G_{r}-S$ and $v_{1}, \ldots, v_{t}$ be fixed elements of $F_{1}, \ldots, F_{t}$ respectively.

Assume first that $v_{1}, \ldots, v_{t}$ are pairwise disjoint. Thus, odd $\left(G_{r}-S\right) \leqslant t \leqslant n / r$. Let $v_{1}=x_{1} \cdots x_{r}$ and $v_{2}=y_{1} \cdots y_{r}$. Then, for all $w_{1}, \ldots, w_{r-2} \in[n]-\left\{v_{1} \cup v_{2}\right\}$ and for all $i, j \in\{1, \ldots, r\}$ the vertices $x_{i} y_{j} w_{1} \cdots w_{r-2}$ are adjacent to both $v_{1}$ and $v_{2}$ and thus must be in $S$. Therefore $|S| \geqslant r^{2}\left(\begin{array}{c}n-2 r \\ r-2\end{array}\right)-1$ (recall that we might have removed one vertex from $\left.V\left(G_{r}\right)\right)$. Easy calculations show that $r^{2}\left(\begin{array}{c}n-2 r \\ r-2\end{array}\right)-1 \geqslant n / r \geqslant \operatorname{odd}\left(G_{r}-S\right)$ as required.

Now suppose that there are $i, j$ with $1 \leqslant i<j \leqslant t$ such that $\left|v_{i} \cap v_{j}\right|=k$ for some $k \in\{2, \ldots, r-1\}$. Without loss of generality let $v_{i}=a_{1} \cdots a_{k} x_{k+1} \cdots x_{r}$ and $v_{j}=a_{1} \cdots a_{k} y_{k+1} \cdots y_{r}$. Then, for all $w_{1} \ldots, w_{r-1} \in[n]-\left\{v_{i} \cup v_{j}\right\}$, the vertices

$$
a_{m} w_{1} \cdots w_{r-1} \text { and } x_{p} y_{q} w_{1} \cdots w_{r-2} \text {, }
$$


where $1 \leqslant m \leqslant k$ and $p, q \in\{k+1, \ldots, r\}$, are adjacent to both $v_{i}$ and $v_{j}$ and thus must be in $S$. Therefore, $|S| \geqslant k\left(\begin{array}{c}n-2 r+k \\ r-1\end{array}\right)+(r-k)^{2}\left(\begin{array}{c}n-2 r+k \\ r-2\end{array}\right)-1$.

It remains to prove that

$$
k\left(\begin{array}{c}
n-2 r+k \\
r-1
\end{array}\right)+(r-k)^{2}\left(\begin{array}{c}
n-2 r+k \\
r-2
\end{array}\right)-1 \geqslant\left(\begin{array}{c}
n \\
r-1
\end{array}\right)
$$

Let $r=3$ and $n \geqslant 12$. We have $k=2$ and (2.3) holds for $n \geqslant 13$. Let us consider the case $n=12$. Then, there is $m \in[t]-\{i, j\}$ such that $v_{m}-\left\{v_{i} \cup v_{j}\right\} \neq \emptyset$. Otherwise, for all $m \in[t]-\{i, j\}$ we have $v_{m} \subseteq v_{i} \cup v_{j}=\left\{a_{1}, a_{2}, x_{3}, y_{3}\right\}$, but then we have only two choices for distinct $v_{m}$ 's which implies $t \leqslant 4$. Let $z \in v_{m}-\left\{v_{i} \cup v_{j}\right\}$. Then, for all $w \in[n]-\left\{v_{i} \cup v_{j} \cup v_{m}\right\}$ the edges $a_{1} z w$ are adjacent to both $v_{i}$ and $v_{m}$ and hence are vertices of $S$. Note that these vertices have not been considered before. Since $\left|[n]-\left\{v_{i} \cup v_{j} \cup v_{m}\right\}\right| \geqslant n-7$ and $n=12$ we have $|S| \geqslant(n-4)^{2}+(n-7)-1 \geqslant\left(\begin{array}{l}n \\ 2\end{array}\right) \geqslant t$.

Now assume that $4 \leqslant r \leqslant \frac{1}{6} \sqrt{n}$, that is, $n \geqslant 576$. We have,

$$
k\left(\begin{array}{c}
n-2 r+k \\
r-1
\end{array}\right)+(r-k)^{2}\left(\begin{array}{c}
n-2 r+k \\
r-2
\end{array}\right) \geqslant 2\left(\begin{array}{c}
n-2 r+k \\
r-1
\end{array}\right) \geqslant 2\left(\begin{array}{c}
n-2 r \\
r-1
\end{array}\right) .
$$

Therefore, to prove (2.3) it is enough to show that

$$
2\left(\begin{array}{c}
n-2 r \\
r-1
\end{array}\right) \geqslant\left(\begin{array}{c}
n \\
r-1
\end{array}\right) \text {. }
$$

Easy calculations show that to prove (2.4) it is enough to show that

$$
\frac{2(n-r+1) \cdots(n-3 r+2)}{n \cdots(n-2 r+1)} \geqslant 1 \text {. }
$$

Observe that the following inequalities hold.

$$
\begin{aligned}
\frac{2(n-r+1) \cdots(n-3 r+2)}{n \cdots(n-2 r+1)} & \geqslant 2\left(\frac{n-3 r+2}{n}\right)^{2 r} \\
& \geqslant 2\left(\frac{n-3 r}{n}\right)^{2 r} \\
& \geqslant 2\left(1-\frac{1}{2 \sqrt{n}}\right)^{2 r} \\
& \geqslant 2\left(1-\frac{1}{2 \sqrt{n}}\right)^{\frac{1}{3} \sqrt{n}}, \quad \text { since } r \leqslant \frac{1}{6} \sqrt{n} .
\end{aligned}
$$

Since $n \geqslant 576$ we have that

$$
2\left(1-\frac{1}{2 \sqrt{n}}\right)^{\frac{1}{3} \sqrt{n}} \geqslant 1
$$

Therefore, inequality (2.5) holds, thus (2.3) is proved. 


\subsection{Domino Decomposition of the Complete 4-graph}

Recall that a domino decomposition of an $r$-graph is a decomposition of its edge set into a fixed $r$-graph with two edges that intersect in exactly two vertices and single edges.

Theorem 2.9. Any optimal domino decomposition of the complete 4-graph with $n \geqslant 6$ vertices has $\left\lceil\frac{1}{2}\left(\begin{array}{l}n \\ 4\end{array}\right)\right\rceil$ elements.

Proof. Clearly any optimal domino decomposition of $K_{n}^{4}$ has at least that many elements. We will now prove by induction that this lower bound is optimal. Let $n=6$. Then

$$
\begin{aligned}
\{\{1234,1256\},\{1235,2456\}, & \{1245,2346\},\{1246,3456\}, \\
\{1345,2356\}, & \{1346,2345\},\{1236,1456\},\{1356\}\}
\end{aligned}
$$

is a domino decomposition of $K_{6}^{4}$. Let $n \geqslant 7$ and assume that the result holds for $n-1$. Let $\mathcal{F}$ be an optimal domino decomposition of $K_{n-1}^{4}$. The result will follow if we show how to extend $\mathcal{F}$ into a domino decomposition of $K_{n}^{4}$ with the prescribed number of elements. Observe that $\mathcal{E}:=E\left(K_{n}^{4}\right)-E\left(K_{n-1}^{4}\right)=\left\{e \cup\{n\} \mid e \in E\left(K_{n-1}^{3}\right)\right\}$ and a domino decomposition of $\mathcal{E}$ is the same as a bowtie decomposition of $K_{n-1}^{3}$. Let $\mathcal{B}$ be an optimal bowtie decomposition of $K_{n-1}^{3}$ and $\mathcal{F}^{\prime}$ its extension to a domino decomposition of $\mathcal{E}$. If $K_{n-1}^{4}$ has an even number of edges then $\mathcal{F} \cup \mathcal{F}^{\prime}$ is clearly a domino decomposition of $K_{n}^{4}$ with the required number of elements. Now suppose that $K_{n-1}^{4}$ has an odd number of edges. In this case $\mathcal{F}$ has an element that is a single edge, say xyzw. If $K_{n-1}^{3}$ has an even number of edges then we are done as before. So suppose that $K_{n-1}^{3}$ has an odd number of edges. Then $\mathcal{B}$ has an element that is a single edge. From the proof of Theorem 2.4 it follows that we can always choose $\mathcal{B}$ such that xya, for some $a \in[n-1]-\{x, y, z, w\}$ is the single edge in $\mathcal{B}$. Then, $\mathcal{F} \cup \mathcal{F}^{\prime} \cup\{x y z w, x y a n\}$ is a domino decomposition of $K_{n}^{4}$ with $\left\lceil\frac{1}{2}\left(\begin{array}{l}n \\ 4\end{array}\right)\right\rceil$ elements.

\subsection{Kite Decompositions of the complete 3-Graph and 4-Graph}

Recall that a kite decomposition of an $r$-graph is a decomposition of its edge set into a fixed $r$-graph with two edges that intersect in exactly $r-1$ vertices and single edges.

Theorem 2.10. Any optimal kite decomposition of the complete 3-graph with $n \geqslant 4$ vertices has $\left\lceil\frac{1}{2}\left(\begin{array}{l}n \\ 3\end{array}\right)\right\rceil$ elements.

Proof. The lower bound follows immediately, thus it suffices to find a kite decomposition of $K_{n}^{3}$ with $\left\lceil\frac{1}{2}\left(\begin{array}{c}n \\ 3\end{array}\right)\right\rceil$ elements. This is done by induction on $n$. If $n=4$ then $\{\{123,234\},\{124,134\}\}$ is a kite decomposition of $K_{4}^{3}$. Let $n \geqslant 5$ and assume that the result holds for $n-1$. Let $\mathcal{F}$ be an optimal kite decomposition of $K_{n-1}^{3}$. The result follows by induction if we show how to extend $\mathcal{F}$ into a kite decomposition of $K_{n}^{3}$ with 
the required number of elements. We first write $E\left(K_{n}^{3}\right)-E\left(K_{n-1}^{3}\right)$ as follows:

$$
\begin{aligned}
& 12 n \quad 13 n \quad 14 n \quad \ldots \quad 1(n-1) n \\
& 23 n \quad 24 n \quad \ldots \quad 2(n-1) n \\
& 34 n \quad \ldots \quad 3(n-1) n \\
& (n-3)(n-2) n \quad(n-3)(n-1) n \\
& (n-2)(n-1) n
\end{aligned}
$$

To proceed we have to consider two different cases.

Case I: Assume that $K_{n-1}^{3}$ has an even number of edges. Then all the elements of $\mathcal{F}$ have two edges, thus it suffices to pair the edges above. This can be done easily by pairing the edges in the same row, starting from left to right, so if a row has an odd number of elements then its element in the last column will be left unpaired. We end up this procedure by pairing the elements left in the last column, leaving exactly one single edge if and only if $K_{n}^{3}$ has an odd number of edges.

Case II: Assume that $K_{n-1}^{3}$ has an odd number of edges. Then $\mathcal{F}$ has an element that is a single edge, say $x y z$ with $x<y<z$. We first pair this edge with the edge $x y n$ and then proceed as in Case I.

Theorem 2.11. Any optimal kite decomposition of the complete 4-graph with $n \geqslant 5$ vertices has $\left\lceil\frac{1}{2}\left(\begin{array}{l}n \\ 4\end{array}\right)\right\rceil$ elements.

Proof. The lower bound follows immediately, thus it suffices to find a kite decomposition of $K_{n}^{4}$ with $\left\lceil\frac{1}{2}\left(\begin{array}{l}n \\ 4\end{array}\right)\right\rceil$ elements. This is done by induction on $n$. If $n=5$ then $\{\{1234,1245\},\{1235,1345\},\{2345\}\}$ is a kite decomposition of $K_{5}^{4}$. Let $n \geqslant 6$ and assume that the result holds for $n-1$. Let $\mathcal{F}$ be an optimal kite decomposition of $K_{n-1}^{4}$. The result follows by induction if we show how to extend $\mathcal{F}$ into a a kite decomposition of $K_{n}^{4}$. We first write $E\left(K_{n}^{4}\right)-E\left(K_{n-1}^{4}\right)$ in $n-3$ groups as follows:

$$
\begin{aligned}
& \begin{array}{llllll}
M_{1}: & 123 n & 124 n & 125 n & \ldots & 12(n-1) n
\end{array} \\
& 134 n 135 n \quad \ldots \quad 13(n-1) n \\
& 1(n-3)(n-2) n \quad 1(n-3)(n-1) n \\
& 1(n-2)(n-1) n
\end{aligned}
$$

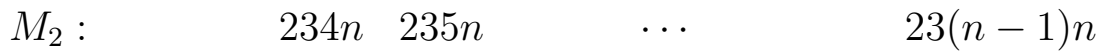

$$
\begin{aligned}
& 245 n \quad \cdots \quad 24(n-1) n \\
& 2(n-3)(n-2) n \quad 2(n-3)(n-1) n \\
& 2(n-2)(n-1) n \\
& M_{n-3}: \quad(n-3)(n-2)(n-1) n
\end{aligned}
$$


To proceed we have to consider two different cases.

Case I: $K_{n-1}^{4}$ has an even number of edges. Then all the elements of $\mathcal{F}$ have two edges, thus it suffices to pair the edges above. This can be done using the following procedure.

For $1 \leqslant i \leqslant n-3$, we consider the group $M_{i}$ and pair the edges in the same row, starting from left to right, so if a row has an odd number of elements then its element in the last column will be left unpaired. We then pair the elements left in the last column starting from top to bottom.

After all groups have been considered all the edges left are of the form $x(n-2)(n-1) n$, with $x \in[n]$. We finish this procedure by pairing these edges, leaving exactly one single edge if and only if $K_{n}^{4}$ has an odd number of edges.

Case II: Assume that $K_{n-1}^{4}$ has an odd number of edges. Then $\mathcal{F}$ has an element that is a single edge, say $x y z w$ with $x<y<z<w$. We first pair this edge with the edge $x y z n$ and then proceed as in Case I.

Acknowledgement. The author thanks Oleg Pikhurko for helpful discussions and comments.

\section{References}

[1] Reinhard Diestel. Graph Theory. Springer-Verlag, 2nd edition, 2000.

[2] P. Erdős. On extremal problems of graphs and generalized graphs. Israel J. Math., 2:183-190, 1964.

[3] Oleg Pikhurko and Teresa Sousa. Minimum $H$-decompositions of graphs. Journal of Combinatorial Theory, B, 97:1041-1055, 2007.

[4] Dijen K. Ray-Chaudhuri and Richard M. Wilson. On t-designs. Osaka J. Math., 12(3):737-744, 1975. 